Classification

Physics Abstracts

16.23

\title{
PRÉPARATION D'ÉCHANTILLONS MÖSSBAUER PAR ÉVAPORATION SOUS VIDE
}

\author{
G. MARCHAL et C. JANOT \\ Laboratoire de Physique du Solide \\ Université de Nancy I, CO no 72 \\ (Ręu le 5 mai 1972, révisé le 17 juillet 1972)
}

\begin{abstract}
Résumé. - On décrit une méthode de préparation d'échantillons susceptibles d'être observés en spectrométrie Mössbauer de transmission. Il s'agit de couches multiples Al-Fe-SiO obtenues par évaporation sous vide, grâce à un dispositif automatique. Trois sources d'évaporation fonctionnent simultanément ; les échantillons se présentent successivement devant chacune d'elles pendant un temps qui dépend de l'épaisseur des dépôts imposée. L'analyse au spectromètre de masse des gaz résiduels pendant les différentes phases de l'opération indique qu'il existe un risque non négligeable de pollution des échantillons par la vapeur d'eau et l'oxyde de carbone, si la vitesse de pompage n'est pas suffisante.

Abstract. - A description is given of a method to obtain vacuum-deposited thin iron films, as monolayers, to be investigated by Mössbauer technique in transmission geometry. Iron is condensed on a thin aluminium film deposited at first, then covered with $\mathrm{SiO}$, then another aluminium film is deposited... and so on to form a number of superposed sandwiches thus increasing the effective absorber thickness. Iron, $\mathrm{SiO}$ and aluminium together are evaporated and the sample « looks at » each cell one after to other during a time depending of the wanted thickness. Residual gazes in the vacuum-room are analysed by mass spectrometer; it appears that corrosion by water molecules or carbon oxyde occurs if the vacuum rate is too weak.
\end{abstract}

I. Introduction. - Une méthode d'approche des états électroniques ou magnétiques de surface consiste à rendre "majoritaires" les atomes superficiels en réalisant des couches très minces du matériau étudié. Plusieurs travaux de ce type ont déjà été effectués par effet Mössbauer [1]. Ce moyen d'observation permet effectivement d'étudier l'environnement immédiat des atomes résonnants. Il est ainsi en principe possible de mesurer l'aimantation de couches très minces et de déterminer les différences qui existent entre atomes de surface et atomes en volume; la méthode est surtout précieuse pour l'approche des propriétés locales comme, par exemple, le moment magnétique porté par des impuretés.

La principale difficulté à vaincre est liée à l'obtention d'échantillons observables en spectrométrie Mössbauer qui nécessite une forte proportion d'atomes concernés par le phénomène étudié. De plus, l'état physico-chimique du matériau (oxydation, pollutions diverses, cristallisation...) doit rester parfaitement contrôlable.

La première partie de ce mémoire décrit la méthode mise au point pour l'élaboration des dépôts très minces de fer. L'obligation de disposer d'une section efficace suffisante en effet Mössbauer suggère une structure d'échantillons en "sandwichs»: une couche épaisse d'aluminium (quelques centaines d'angströms), un dépôt très mince de fer (de l'ordre de l'angström), une couche d'isolant $\mathrm{SiO}$ entre chaque doublet $\mathrm{Al}-\mathrm{Fe}$ (une centaine d'angströms), etc. Un calcul rapide montre que 100 à 200 couches atomiques de fer sont nécessaires si l'on opère avec du fer enrichi en isotope ${ }^{57} \mathrm{Fe}$ et plusieurs milliers de couches atomiques si l'on dépose du fer naturel.

La nécessité d'éviter absolument la pollution, en particulier la pollution par l'oxygène et la vapeur d'eau qui fixent les moments magnétiques dans les oxydes ou les hydroxydes de fer, implique une analyse des gaz résiduels pendant les évaporations; la seconde partie de ce travail révèle les résultats de cette analyse et indique les contraintes de travail qui en découlent.

II. Description du dispositif d'évaporation. - Les couches minces $\mathrm{Al}-\mathrm{Fe}-\mathrm{SiO}$ sont déposées par évaporation thermique dans une enceinte classique (Veeco VE-770). Le vide limite de l'enceinte, équipée du dispositif d'évaporation, est $10^{-7}$ torr. Durant les évaporations, la pression varie entre $10^{-5}$ et $10^{-6}$ torr selon les conditions opératoires.

La réalisation de plusieurs milliers de «sandwichs » $\mathrm{Al}-\mathrm{Fe}-\mathrm{SiO}$ dans un temps raisonnable (quelques heures) et la récupération des produits déposés ont déterminé les caractéristiques du dispositif d'évaporation.

II.1. STRUCTURE D'ENSEMble DU DISPOSITIF D'ÉVAPORATION. - La réduction de la durée des manipulations peut être obtenue en agissant d'une part sur la 
géométrie du dispositif et d'autre part sur la vitesse d'évaporation des sources. Ceci suggère : une optimisation de la distance source-substrat et de l'angle solide sous lequel la source voit les substrats, un fonctionnement simultané des 3 sources d'évaporation avec dispositif de séparation des faisceaux atomiques.

Dans le dispositif retenu, les substrats mobiles peuvent se présenter successivement devant chaque source d'évaporation, ce qui élimine la présence de caches mobiles mais réduit le nombre d'échantillons disponibles et rend difficile le contrôle de la température des substrats. Ce dispositif permet en outre une réduction sensible de la quantité de fer totale nécessaire et diminue la durée des manipulations grâce à l'utilisation de masques à ouverture variable.

Le dispositif d'évaporation (Fig. 1) comprend : la tourelle porte-substrats, la platine de masques à

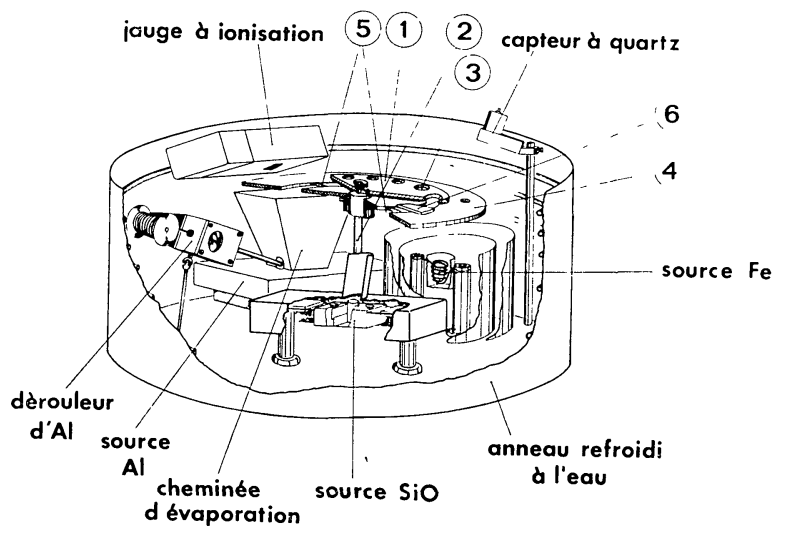

FIG. 1. - Vue d'ensemble du dispositif d'évaporation.

ouverture variable, les 3 sources d'évaporation surmontées de leurs cheminées, les capteurs de vitesses d'évaporation. L'échantillon, animé d'un mouvement circulaire uniforme, se présente successivement devant chacune des 3 sources d'évaporation. On réalise ainsi un empilement de triplets $\mathrm{Al}-\mathrm{Fe}-\mathrm{SiO} . . . \mathrm{Al}-\mathrm{Fe}-\mathrm{SiO} . .$. , etc. dont les épaisseurs sont déterminées par le produit de la vitesse de dépôt par le temps de séjour de l'échantillon devant chaque source.

II.2. La tOURELle PORTE-SUbSTRATS. - C'est un disque épais en acier inoxydable (1) fixé à une extrémité de l'axe de rotation (2) ; l'autre extrémité de cet axe est couplée à un moteur d'entraînement (vitesse réglable entre 0,1 et 10 tours $/ \mathrm{mn}$ ) par l'intermédiaire d'un passage tournant. Ce disque est percé d'ouvertures circulaires (diamètre $20 \mathrm{~mm}$ (3)) destinées à recevoir les substrats. Ces ouvertures sont disposées sur une couronne circulaire dont le diamètre a été déterminé en tenant compte du nombre d'échantillons nécessaires, de la dimension du diamètre du cercle sur lequel sont disposées les 3 sources d'évaporation et de l'angle solide sous lequel les échantillons sont vus depuis les sources, afin d'obtenir des dépôts d'épaisseur homogène sur un diamètre utile de $10 \mathrm{~mm}$. Ces contraintes réduisent à 12 le nombre de substrats; ils sont disposés tangentiellement sur un même cercle et le disque porte-substrats est à $100 \mathrm{~mm}$ au-dessus des 3 sources d'évaporation. Les substrats sont des disques d'épaisseur comprise entre 10 et $40 \mu \mathrm{m}$ découpés dans des feuilles d'aluminium très pur obtenues après laminage d'un barreau d'aluminium purifié au Laboratoire par 20 passes de fusion de zone ( 5 p. p. m. d'impuretés). Avant leur mise en place les substrats subissent une préparation classique de l'état de surface.

II.3. La Platine PoRte-MASQues. - Placée entre les sources d'évaporation et les substrats, elle définit l'aire de dépôt sur les substrats ; dans le cas d'absorbeurs Mössbauer, cette aire demeure identique pour les trois matériaux déposés. Ceci implique la réalisation d'une platine porte-masques (4), centrée sur l'axe de rotation des substrats et dans laquelle ont été découpées 3 ouvertures (5) (une en face de chaque source d'évaporation). Ces ouvertures sont des portions de secteurs circulaires obturables à l'aide de secteurs mobiles (6). Ces secteurs mobiles permettent de régler l'ouverture, donc le temps d'exposition d'un échantillon au-dessus de chaque source. Il est ainsi possible d'atteindre un rapport 1000 entre les épaisseurs d'aluminium et de fer, rapport qui ne pourrait être obtenu en agissant uniquement sur les températures des sources d'évaporation.

II.4. LeS SOURCES D'ÉvAPORATION. - Les caractéristiques géométriques du système d'évaporation étant déterminées, la nature du substrat connue, le seul paramètre disponible pour régler l'épaisseur des dépôts est la vitesse d'évaporation des sources. Les vitesses d'évaporation sont reliées aux pressions de vapeur des métaux à évaporer [2], à la température des sources et à la surface d'évaporation. Compte tenu des vitesses de dépôt nécessaires $(5 \AA / \mathrm{s}$ pour le fer et $500 \AA / \mathrm{s}$ pour l'aluminium), nous avons été amenés à réaliser 2 sources d'évaporation ayant les caractéristiques suivantes : pour la source d'aluminium, température de source voisine de $1300^{\circ} \mathrm{C}$, surface d'évaporation $3 \mathrm{~cm}^{2}$; pour la source de fer, température de source voisine de $1500^{\circ} \mathrm{C}$, surface d'évaporation $0,5 \mathrm{~cm}^{2}$. La source de sublimation de $\mathrm{SiO}$ est un creuset Veeco en tantale chauffé par effet Joule. Ce creuset possède un triple baffle optique éliminant le départ de particules solides et des écrans de rayonnement évitant un chauffage excessif des substrats et des parois de l'enceinte. Après dégazage progressif du creuset rempli de $\mathrm{SiO}$ (Union Carbide, select grade) pendant une durée de 2 heures, sa température est maintenue vers $1200^{\circ} \mathrm{C}$ en réglant manuellement l'intensité du courant qui le traverse. On obtient ainsi des vitesses de dépôt voisines de $100 \AA / \mathrm{s}$ au niveau des substrats.

La source de sublimation-évaporation de fer est une source à chauffage indirect. Elle est constituée d'un creuset cylindrique en alumine (Degussa, AL 23) de volume utile $0,5 \mathrm{~cm}^{3}$ contenant le fer en morceaux 
et muni d'écrans thermiques concentriques en tantale. Ce creuset est chauffé par un enroulement en tungstène multibrins spiralé autour de l'alumine. Les vitesses de dépôt sont comprises entre 1 et $10 \AA / \mathrm{s}$ au niveau des substrats. Il a fallu renoncer à l'emploi de creusets en métaux réfractaires recouverts d'alumine car ces derniers sont rapidement détruits par formation d'alliages avec le fer à travers la couche protectrice d'alumine.

La source d'évaporation d'aluminium (Veeco SC 007) est une source à chauffage indirect alimentée en continu (Fig. 2). C'est un creuset en nitrure de

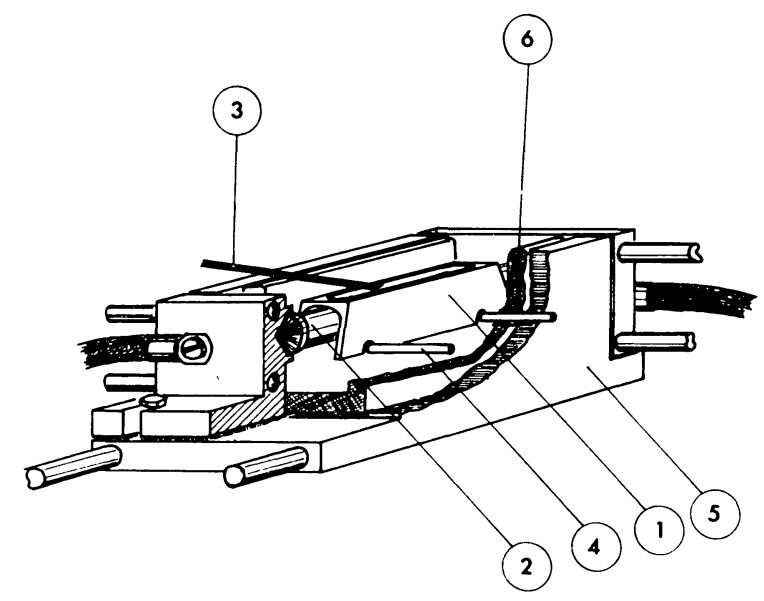

FIG. 2. - Source d'évaporation en continu de l'aluminium. La cheminée d'évaporation n'est pas représentée.

bore (1) (Carborundum, grade A) chauffé par le rayonnement d'un barreau cylindrique en graphite (2) (Carbone Lorraine, 5890/PT), lui-même chauffé par effet Joule. Le fil d'aluminium (3) (Semi-Eléments Inc., grade $5 \mathrm{~N}$ ) est amené dans le creuset en nitrure de bore à l'aide d'un dérouleur entraîné par un moteur à vitesse variable. Ce creuset est supporté par 4 tétons (4) fixés dans un bloc en cuivre (5) refroidi à l'eau. Des écrans thermiques en feutre de graphite (6). isolent le creuset des parois refroidies. La vitesse d'évaporation de l'aluminium est réglée en agissant sur la température du barreau de graphite et sur la vitesse d'entrâ̂nement $\mathrm{du}$ fil d'aluminium. Des vitesses de dépôt de $500 \AA / \mathrm{s}$ sont facilement obtenues au niveau des substrats ; 4 évaporations de 3 heures chacune correspondant à des empilements de 2000 à 3000 sandwichs sont réalisées avant qu'il soit nécessaire de changer l'ensemble barreau de graphite et creuset de nitrure de bore. La seconde partie de ce travail montrera cependant que cette source est le siège d'un dégagement important d'oxyde de carbone provenant du barreau de graphite. Dans sa nouvelle version, l'ensemble barreau de graphite-creuset de nitrure de bore sera remplacé par un creuset intermétallique en nitrure de bore-diborure de titane, à chauffage direct.

Des cheminées, placées au-dessus de chaque source d'évaporation, limitent le dépôt des matériaux sur les parois de l'enceinte, ce qui élimine tout nettoyage de celle-ci et permet une récupération du fer par attaque chimique, lorsqu'on utilise du ${ }^{57} \mathrm{Fe}$. Il s'agit de cheminées doubles à symétrie verticale, une gaine étant dirigée vers les échantillons et l'autre vers les capteurs de vitesses d'évaporation.

II. 5 LES CAPTEURS DE VITESSES D'ÉVAPORATION. Durant l'évaporation, l'épaisseur des dépôts de fer est mesurée à l'aide d'un capteur à quartz. En ce qui concerne l'aluminium, les quantités de matériau évaporé sont trop importantes pour qu'un dispositif à quartz puisse être employé et il a semblé préférable d'utiliser une jauge à ionisation non saturable type Noralp. Un détecteur synchrone fournit un signal directement proportionnel à la densité de vapeur; les épaisseurs peuvent être lues après intégration de ce signal. Le contrôle des dépôts de $\mathrm{SiO}$ n'ayant pas à être aussi rigoureux, la régularité approximative des épaisseurs est obtenue par la reproductibilité des temps d'exposition devant la source d'évaporation préalablement étalonnée.

\section{Nature des gaz résiduels durant l'évaporation} de couches minces Al-Fe-SiO. - III. 1. INTRODUCTION. - Il est primordial d'évaluer les risques de pollution des matériaux évaporés par l'atmosphère résiduelle. Dans des conditions normales d'évaporation, la probabilité de collision entre les molécules ou atomes métalliques s'évaporant et les molécules d'oxygène résiduel en transit entre source et substrat est très faible. Cependant, le substrat est bombardé par un relativement grand nombre de molécules gazeuses de sorte qu'il y a une probabilité plus élevée d'oxydation après dépôt. Il peut également y avoir formation d'oxydes au niveau de la source et évaporation de ces oxydes. Si l'on calcule le rapport du nombre d'impact sur les substrats des molécules gazeuses d'oxygène à celui des atomes de vapeur métallique dans des conditions voisines de celles rencontrées durant les évaporations et en considérant que la pression d'oxygène est du même ordre de grandeur que la pression totale, on obtient un effet négligeable pour l'aluminium (rapport de l'ordre de $10^{-2}$ ) mais un risque certain de pollution dans le cas du fer (rapport voisin de 1).

Il est donc important de connaître la nature des gaz résiduels présents dans l'enceinte d'évaporation durant la réalisation de multicouches $\mathrm{Al}-\mathrm{Fe}-\mathrm{SiO}$, pour en déduire les contraintes expérimentales impératives, sur la qualité du vide notamment.

III. 2. Conditions D'ANALYSE. - Un spectromètre de masse Veeco, type Monopole, a donc été monté en parallèle avec l'enceinte d'évaporation pour examiner la nature des gaz résiduels présents avant, durant et après l'évaporation des 3 matériaux ( $\mathrm{Al}, \mathrm{Fe}, \mathrm{SiO})$. Le balayage des masses 1 à 45 , effectué en $4 \mathrm{mn}$, donne une réduction de la hauteur des pics d'environ $5 \%$ par rapport à l'équilibre. Deux balayages successifs sont nécessaires pour obtenir une bonne précision des intensités des pics correspondant aux éléments 
peu représentés. Les pourcentages approximatifs des principaux constituants gazeux de l'enceinte ont été obtenus à partir des intensités des courants d'ions mesurés, en négligeant les ions de masse supérieurs à 45. L'analyse d'un mélange gazeux tel que celui de l'atmosphère résiduelle d'une enceinte d'évaporation ne peut être faite sans la connaissance des réseaux de cracking des composés gazeux. Nous avons utilisé ici le tableau de fragmentation des gaz résiduels obtenu par Klopper et Schmidt [3] à l'aide d'un omégatron modifié. Il est également possible d'identifier des espèces de poids moléculaire élevé $(m / e>50)$ en observant le réseau de cracking dans l'intervalle des masses 1 à 50. Ainsi, les ions fragments d'hydrocarbures caractéristiques des huiles de pompes primaires et secondaires ont été répertoriés pour permettre l'identification de la rétrodiffusion et de la rétromigration des huiles et les séparer ainsi des autres sources de contamination de l'enceinte d'évaporation. L'observation du réseau de cracking [4] de ces hydrocarbures montre que la présence de ceux-ci peut être reconnue par les ions de $m / e=41,43,55,57 \ldots$, etc. et leur concentration relative estimée à partir de l'ion $m / e=43$ (radical $\mathrm{CH}_{3} \mathrm{CH}_{2} \mathrm{CH}_{2}^{+}$) présent dans tous les crackings d'hydrocarbures contenant au moins 4 atomes de carbone. Les huiles silicones (par exemple DC 704) utilisées dans les pompes à diffusion constituent une seconde source de molécules à poids moléculaire élevé. Malheureusement les réseaux de cracking de ces huiles semblent être difficilement reproductibles [5]-[6]. Toutefois, la présence de ces huiles dans l'enceinte peut être décelée par l'apparition de pics à $m / e=43,51,55,57, \ldots$ normalement absents dans une enceinte propre. La présence de traces d'huiles DC 704 dans l'enceinte entraîne également l'apparition de pics à $m / e=28,32,44$ pics rencontrés fréquemment dans une enceinte non dégazée.

Les spectres de masse des gaz résiduels de l'enceinte d'évaporation ont été enregistrés à différents intervalles correspondant aux processus classiques d'une évaporation : avant l'évaporation, durant le dégazage des sources, pendant l'évaporation et après cette évaporation. Les courants d'ions ont été reportés sur 4 diagrammes (I, II, III, IV) correspondant à ces différents stades de l'évaporation. Ces stades ont des durées différentes selon le matériau et la source utilisés. Les conditions expérimentales ont été les suivantes:

- la première série (diagramme I) concerne les spectres réalisés pendant, l'évaporation du fer (vitesse de dépôt sur les substrats $6,5 \AA / \mathrm{s}$, durée de l'évaporation $3200 \mathrm{~s}$ );

- la seconde série (diagramme II) est réalisée, après remise à l'air de l'enceinte, pendant l'évaporation de $\mathrm{SiO}$ (vitesse de dépôt de $\mathrm{SiO}$ sur les substrats $100 \AA /$ s, durée de l'évaporation 3000 s) ;

- la troisième série (diagramme III) se rapporte à l'évaporation d'aluminium après remise à l'air de l'enceinte (vitesse de dépôt d'aluminium sur les substrats $200 \AA / \mathrm{s}$, durée de l'évaporation 1800 s) ;
- la dernière série (diagramme IV) est enregistrée pendant le dépôt simultané des' 3 matériaux $\mathrm{Al}, \mathrm{Fe}, \mathrm{SiO}$ dans les conditions précédentes (durée de l'évaporation $1200 \mathrm{~s}$ ).

Un balayage à l'azote est effectué après chaque remise à l'air de l'enceinte.

III. 3 ETUDE DE LA COMPOSITION DES GAZ RÉSIDUELS DE L'ENCEINTE AVANT LE DÉGAZAGE DES SOURCES D'ÉVAPORATION. - Les spectres 1 des 4 diagrammes concernent l'atmosphère résiduelle de l'enceinte d'évaporation évacuée par la pompe à diffusion d'huile (DC 704) et isolée par un piège à chevrons refroidis à l'eau, sans piège à azote liquide. L'atmosphère résiduelle est composée de différents gaz produisant des courants ioniques dans le spectromètre à différentes valeurs de $m / e$.

A l'aide du tableau de Klopfer et Schmidt [3], les ions de $m / e=17,18$ peuvent être attribués à la vapeur d'eau qui représente $76 \%$ des gaz résiduels de l'enceinte non dégazée et non piégée. Ensuite viennent les ions $m / e=28(10 \%), m / e=44 \quad(4 \%)$ et $m / e=2(3 \%)$. Le rapport des intensités des pics de $m / e=14,28$ semble indiquer que le pic de $m / e=28$ n'est pas attribuable uniquement à l'azote. La présence d'hydrocarbures est mise en évidence par les ions de $m / e=27,29,41,43$ dont les variations des courants d'ions de $m / e=27$ et 43 ont été représentées sur les diagrammes III et IV.

Lorsqu'on remplit d'air liquide le piège situé audessus de la pompe à diffusion d'huile, on constate une diminution de l'amplitude des différents pics (spectre 2). Or, ce piège n'agit que sur la vapeur d'eau et le gaz carbonique. Cependant, on observe également une diminution de l'amplitude des pics correspondant à des ions provenant de gaz non condensables à cette température. Ceci permet d'affirmer que l'ion $m / e=32$ est dû à la fois à l'oxygène résiduel et à $\mathrm{CO}_{2}$ alors que $m / e=28$ est dû essentiellement à l'oxyde de carbone.

Afin de réduire le dégazage des parois de l'enceinte causé par le rayonnement des sources, un anneau en cuivre refroidi à l'eau a été monté à l'intérieur de la cloche en pyrex (Fig. 1); d'autre part, la source d'évaporation d'aluminium possède des parois en cuivre refroidies à l'eau (Fig. 2). Les raccords d'étanchéité d'eau métal-plastique présentent une légère fuite, comme on peut le constater en comparant les spectres 3 aux spectres 1 et 2 obtenus en l'absence de refroidissement. Dans ces conditions, la pression partielle de vapeur d'eau (ions de $m / e=1,17,18$ ) atteint $80 \%$ de la pression totale. Les autres courants d'ions demeurent inchangés : $m / e=2$ (hydrogène) $3 \%$, $m / e=28$ (azote + monoxyde de carbone) $5 \%$, $m / e=44\left(\mathrm{CO}_{2}\right) 2 \%$.

III. 4 ETUde DU DÉGAZAGE DE LA SOURCE DE FER. Les variations des pressions partielles des principaux gaz sont rassemblées figure 3 sur le diagramme I 


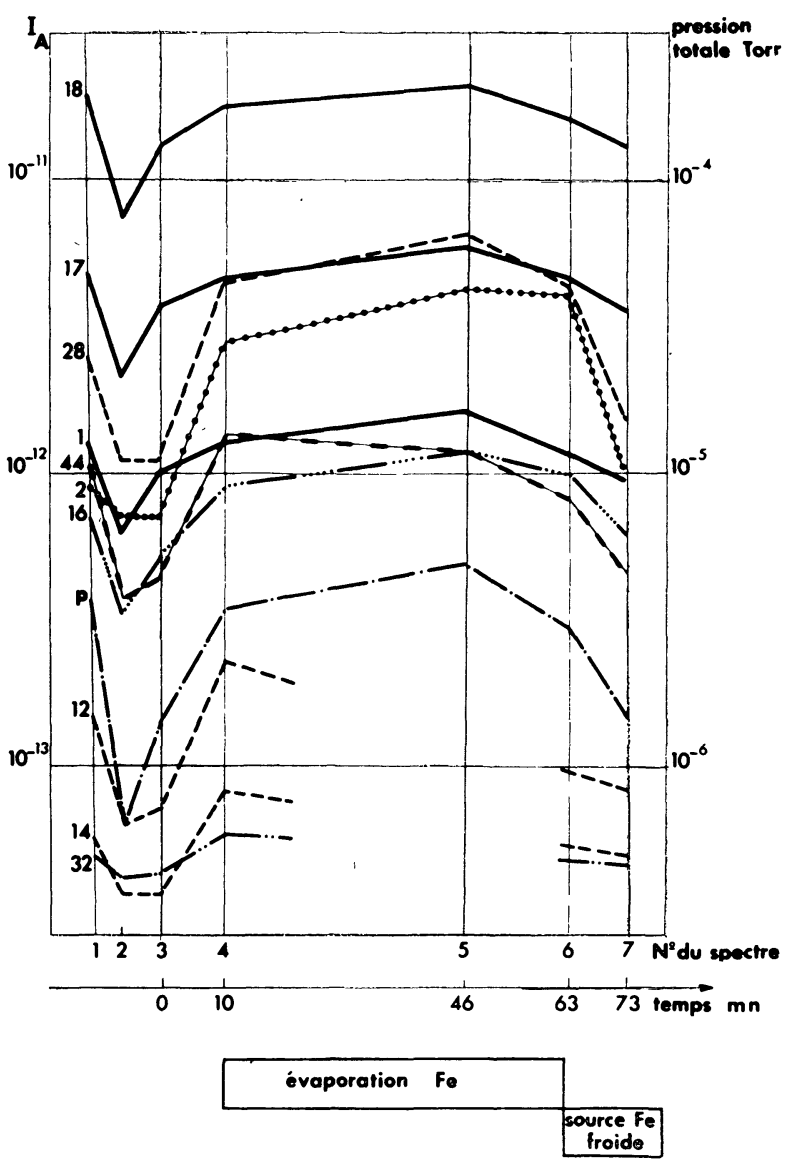

FIG. 3. - Variation des pressions partielles et de la pression totale en fonction du temps durant l'évaporation de fer. Chaque courbe est repérée par le nombre de masse correspondant.

(spectres 4 à 7). L'observation de ce diagramme montre deux séries de variations dans le temps :

- la pression partielle de vapeur d'eau $(m / e=1$, $17,18)$ augmente puis diminue ensuite. Cette variation est liée au dégazage des écrans thermiques de la source et des parois de l'enceinte. Lorsque la source a atteint sa température de fonctionnement, le dégazage diminue dans le temps ;

- les ions de $m / e=2,12,14$ et 28 donnent des pics dont les amplitudes subissent la même loi de variation dans le temps. Ces ions proviennent des gaz libérés par la source de fer (désorption de la surface des écrans et diffusion des gaz occlus dans le fer).

Pendant l'évaporation du fer, la composition de l'atmosphère résiduelle est la suivante : $60 \% \mathrm{de}$ vapeur d'eau, $12 \%$ d'oxyde de carbone et $8,2 \%$ d'hydrogène. La pression partielle d'oxygène demeure toujours très faible, elle est toutefois présente en quantités non négligeables sous forme d'ions $\mathrm{CO}, \mathrm{OH}$.

Ces résultats peuvent être comparés à ceux obtenus par Wagener et Marth [7] lors de l'étude au spectromètre de masse des gaz libérés par chauffage de fer à $1000^{\circ} \mathrm{C}$. Ces auteurs ont trouvé une composition de $95 \%$ d'oxyde de carbone, $3 \%$ de vapeur d'eau et $2 \%$ d'azote. Deux différences apparaissent : absence d'hydrogène et faible proportion de vapeur d'eau. L'absence d'hydrogène semble anormale, puisque ce gaz diffuse facilement dans le fer et se rassemble aux joints de grains, mais peut s'expliquer par les origines différentes des matériaux étudiés. Par contre, la forte proportion de vapeur d'eau présente ici durant l'évaporation de fer provient essentiellement de l'atmosphère résiduelle de l'enceinte.

Durant le dégazage de la source de fer, puis l'évaporation de fer, on constate un dégagement important d'hydrogène et d'oxyde de carbone. La pression partielle de vapeur d'eau varie moins rapidement et diminue même en fin d'évaporation. Cet abaissement de la pression de $\mathrm{H}_{2} \mathrm{O}$ peut s'expliquer par l'action $\mathrm{du}$ piège à azote liquide et par une réaction possible du fer sur la vapeur d'eau. L'oxyde de carbone provient de la réduction de l'oxyde de fer par le carbone présent comme impureté et il a comme ion fragment $\mathrm{m} / e=12$ comme le montrent les variations du spectre correspondant.

III.5 ANALYSE PENDANT LE DÉGAZAGE ET L'ÉVAPORATION DE SiO. - Les variations des pressions partielles des principaux constituants de l'atmosphère résiduelle de l'enceinte sont représentées figure 4 (diagramme II, spectres 4-14). Les différentes étapes de l'évaporation sont notées en face de chaque spectre

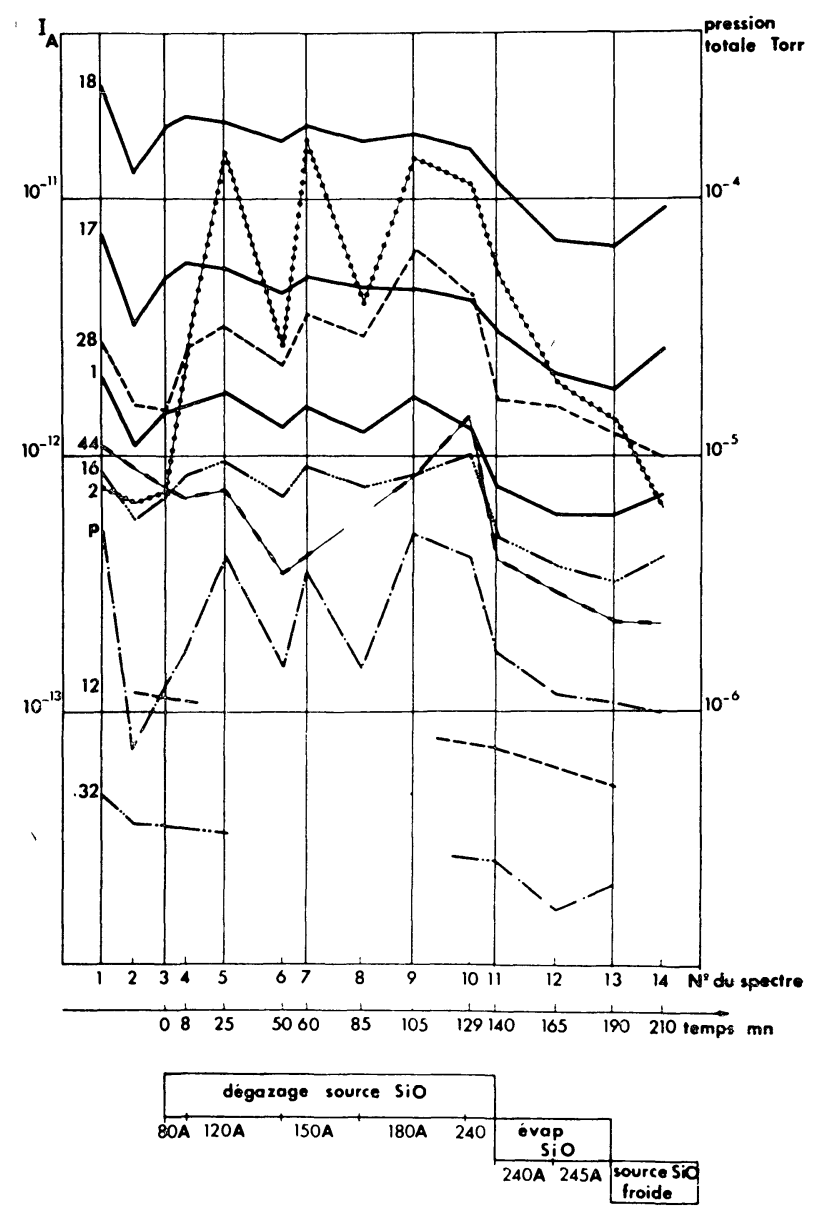

FIG. 4. - Evolution des pressions partielles et de la pression totale en fonction du temps durant la sublimation de $\mathrm{SiO}$. 


\section{TABLEAU I}

\section{Avant \\ l'évaporation \\ Gaz résiduels}$$
\text { - }
$$

Eau $m / e ~ 1,17,18$

Hydrogène $m / e 2$

Oxyde de carbone $m / e 28$

Méthane $m / e 16$

Oxygène $m / e 32$

Gaz carbonique $m / e$

$m / e 12,14,15$

en détaillant plus particulièrement le dégazage de la poudre, effectué par paliers successifs. Par exemple, en fixant l'intensité du courant qui circule dans le creuset à $120 \mathrm{~A}$, on constate une élévation de la pression totale que l'on retrouve sur le spectre 5 , puis dès que cette pression est revenue à sa valeur initiale (spectre 6), l'intensité du courant est élevée à $150 \mathrm{~A}$. Le dégazage est ainsi effectué progressivement pour éviter d'une part des projections de poudre hors du creuset et d'autre part afin de limiter les remontées importantes de pression ( $\mathrm{p}_{\text {totale }}<2 \times 10^{-5}$ torr).

L'observation du diagramme II montre trois phénomènes très distincts :

- une variation très forte de la pression partielle d'hydrogène $(m / e=2)$. Celle-ci subit une variation en dents de scie correspondant aux étapes successives du dégazage puis une chute très rapide dès le début de l'évaporation de $\mathrm{SiO}$;

- une augmentation très limitée de la pression partielle de vapeur d'eau $(m / e=1,17,18)$ au début de l'échauffement du creuset, suivie par une baisse régulière et constante durant l'évaporation ;

- dès l'arrêt de l'évaporation (spectre 13), la pression partielle de la vapeur d'eau $(m / e=1,17,18)$ remonte très rapidement.

Les variations de la composition de l'atmosphère résiduelle au cours des quatre stades de l'évaporation sont résumées tableau I. Ce tableau montre que, avant l'évaporation, la vapeur d'eau constitue le gaz résiduel principal mais que, durant l'évaporation et surtout pendant le dégazage de la source, l'hydrogène libéré par cette source (creuset de tantale) représente une fraction élevée de la pression totale [8]. Enfin, après l'évaporation, on retrouve approximativement la composition de départ, avec un léger déficit en vapeur d'eau.

Enfin, il faut noter l'effet getter très important de $\mathrm{SiO}$ sur $\mathrm{H}_{2} \mathrm{O}$ et $\mathrm{O}_{2}$ [9], [10], [11]. Les pressions partielles de vapeur d'eau sont réduites dans un rapport 3 et celles d'oxygène dans un rapport 2 entre le début du dégazage de la source (spectre 3 ) et la fin de l'évaporation (spectre 13) alors que la pression totale a conservé sa valeur de départ.
Composition de l'atmosphère résiduelle $(\%)$

$\begin{array}{ccc}\begin{array}{c}\text { Pendant le } \\ \text { dégazage } \\ \text { Spectre } 7\end{array} & \begin{array}{c}\text { Durant } \\ \text { l'évaporation } \\ \text { Spectre } 12\end{array} & \begin{array}{c}\text { Après } \\ \text { l'évaporation } \\ \text { Spectre } 14\end{array} \\ 53 & - & - \\ 35 & 68 & 81 \\ 7,5 & 14,5 & 4,2 \\ 1,8 & 11 & 6,2 \\ 0,07 & 2,5 & 2,8 \\ 0,9 & 0,1 & \text { non mesuré } \\ 0,3 & 2 & 1,4 \\ & 1 & \text { non mesuré }\end{array}$

III. 6 ANALYSE PENDANT LE DÉGAZAGE ET L'ÉVAPORATION D'ALUMINIUM. - Le diagramme III (Fig. 5) représente l'évolution des pressions partielles des différents gaz dans l'enceinte pendant le dégazage

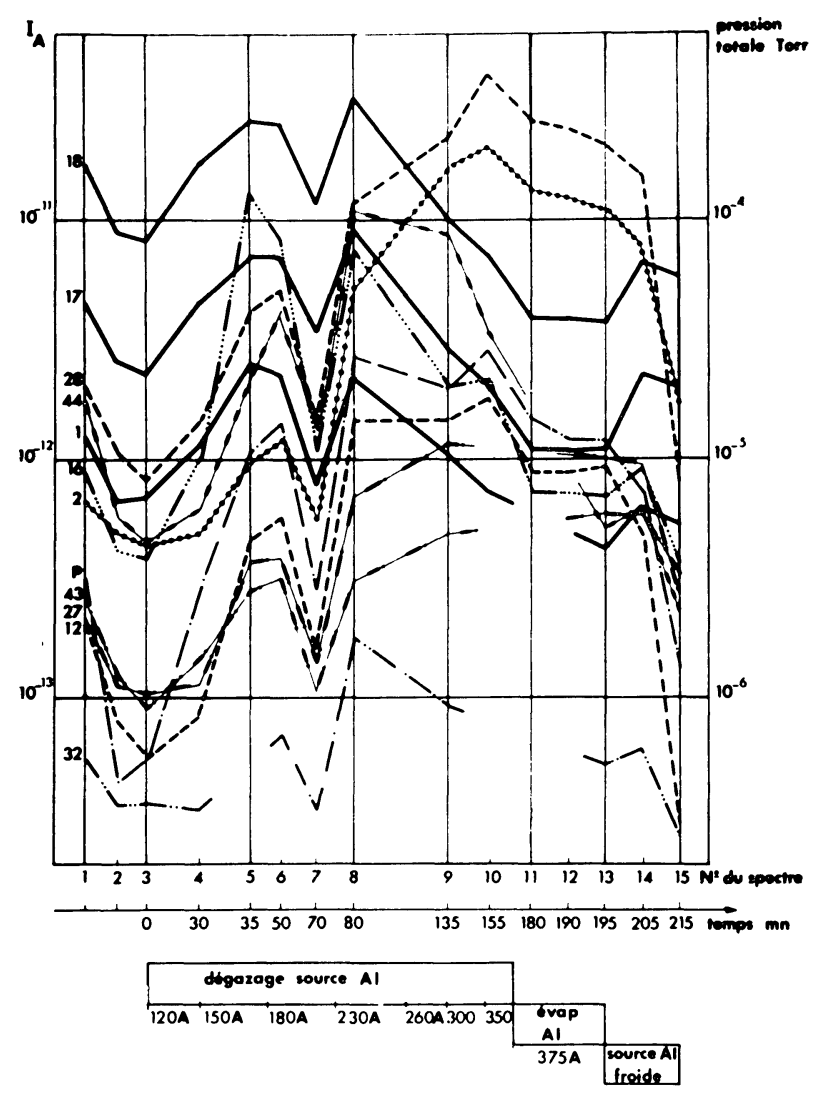

FIG. 5. - Variation des pressions partielles et de la pression totale en fonction du temps durant l'évaporation d'aluminium.

puis l'évaporation d'aluminium. A partir de ce diagramme, la composition de l'atmosphère résiduelle de l'enceinte a été déterminée (Tableau II) :

- avant le dégazage de la source,

- durant le dégazage de la source vide,

- à la fin du dégazage,

— pendant l'évaporation d'aluminium. 
TABLEAU II

\section{Gaz résiduels}

Eau $m / e$ 1, 17, 18

Hydrogène $m / e 2$

Oxyde de carbone + azote $m / e 28$

Oxygène $m / e 32$

Gaz carbonique $m / e 44$

Méthane $m / e 16,15$

Hydrocarbure $m / e$ 27, 43, 45
Composition de l'atmosphère résiduelle $(\%)$
Avant dégazage 76

3

5,5

0,2

3

3

2,5

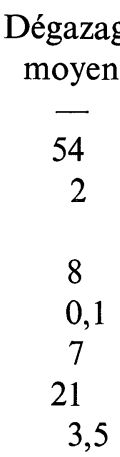

Fin du
dégazage

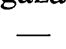

20

24

30

0,1

13

4

3
Pendant

évaporation

L'interprétation de ces résultats nécessite la connaissance des sources de gaz libérés. Ceux-ci peuvent provenir du dégazage des différentes parties de la source (Fig. 2) :

- barreau de graphite,

- creuset en nitrure de bore,

- écrans de rayonnement et parois de l'enceinte,

- joints d'étanchéité et cracking d'hydrocarbures au contact de la source,

- enfin, métal lui-même.

L'examen du diagramme III et du tableau II qui en découle joint à la liste des sources de dégazage possibles énoncées précédemment conduit aux remarques suivantes :

a) Les masses $1,17,18$ suivent une évolution semblable durant tout le cycle. Ces masses caractérisent la vapeur d'eau. On note une élévation de ces pressions partielles dès le début du chauffage puis une diminution avant la fin du dégazage de la source ; la vapeur d'eau est libérée à des températures inférieures à $500^{\circ} \mathrm{C}$. Cette diminution des pressions partielles de vapeur d'eau est encore accentuée dès le début de l'évapora. tion d'aluminium. Le pourcentage de vapeur d'eau qui était de $76 \%$ avant le dégazage tombe à $12 \%$ durant l'évaporation. Comme pour l'évaporation de $\mathrm{SiO}$, la vapeur d'eau est getterisée par l'aluminium $\left(2 \mathrm{Al}+3 \mathrm{H}_{2} \mathrm{O} \rightarrow \mathrm{Al}_{2} \mathrm{O}_{3}+3 \mathrm{H}_{2}\right)$. C'est là le phénomène le plus important.

L'hydrogène libéré par une telle réaction est pompé plus rapidement que la vapeur d'eau par la pompe à diffusion et la pression totale diminue. Dès que l'évaporation d'aluminium cesse (spectre 13) on assiste à une remontée des pressions partielles de vapeur d'eau.

b) L'hydrogène $(m / e=2)$ provient de la décomposition de la vapeur d'eau getterisée par l'aluminium et du dégazage du barreau de graphite.

c) La masse $m=28$ peut être attribuée essentiellement à l'oxyde de carbone émis par le graphite [12], [13].

III.7 ETUdE DE L'ÉVAPORATION SIMULTANÉE Al-Fe-SiO. — Le diagramme IV (Fig. 6) présente

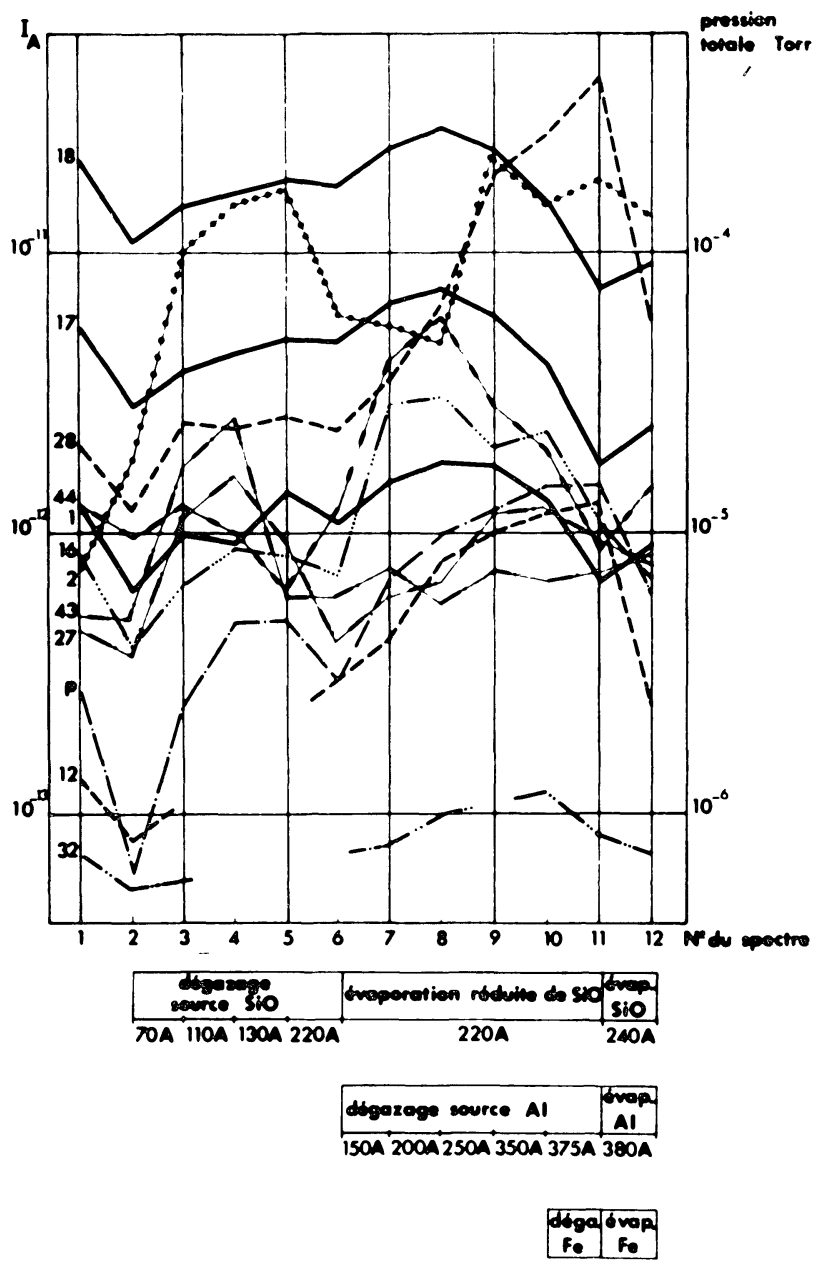

FIG. 6. - Evolution des pressions partielles et de la pression totale pendant l'évaporation simultanée $\mathrm{Al}-\mathrm{Fe}-\mathrm{SiO}$.

l'évolution des pressions partielles durant le dégazage des sources puis l'évaporation simultanée Al-Fe-SiO. L'ordre des dégazages des sources est déterminé par les durées respectives de ces opérations :

a) La source de $\mathrm{SiO}$ demande un dégazage de plusieurs heures ; c'est donc elle qui est dégazée en premier. On retrouve (diagramme IV) un fort dégagement d'hydrogène (spectres 2 à 5) qui s'atténue dès que la 


\section{TABLEAU III}

Composition de l'atmosphère résiduelle $(\%)$

Evaporation $\mathrm{Al}$
seul
Spectre 13
Diagramme III
-

12

25

48

Hydrogène $m / e 2$

Oxyde de carbone $m / e 28$

Oxygène $m / e 32$

Gaz carbonique $m / e 44$

Méthane $m / e 15,16$

Hydrocarbures $m / e$ 27, 43, 45

Evaporation
$\mathrm{Al}+\mathrm{SiO}$
Spectre 11
Diagramme IV

12

22

52

0,1

1

2

2,5
Evaporation

$\mathrm{Al}+\mathrm{Fe}+\mathrm{SiO}$

Spectre 12

Diagramme IV source a atteint la température requise pour l'évaporation (spectre 6). La variation de la pression partielle d'hydrogène détermine celle de la pression totale. Les autres pics qui évoluent sont ceux des hydrocarbures $(m / e$ 27, 43) libérés par le joint d'étanchéité de la cloche en pyrex chauffée par le rayonnement du creuset de $\mathrm{SiO}$.

b) Lorsque le dégazage de la source de $\mathrm{SiO}$ est terminé, on commence celui de la source d'aluminium ( $1 \mathrm{~h}$ environ) tout en maintenant la température de la source de $\mathrm{SiO}$ à une valeur légèrement inférieure à celle requise pour une évaporation normale. On retrouve, comme sur le diagramme III, le départ successif des différents gaz libérés par la source d'aluminium : d'abord le méthane et le gaz carbonique (spectres 6 à 8) puis l'hydrogène (spectre 9) et l'oxyde de carbone (spectres 8 à 11).

Lorsque la température est suffisamment élevée, l'évaporation de l'aluminium est déclenchée (spectre 11). On notera que la composition des gaz résiduels durant l'évaporation simultanée d'aluminium et de $\mathrm{SiO}$ (spectre 11, diagramme IV) est identique à celle correspondant à l'évaporation d'aluminium seule (spectre 13, diagramme III). Les résultats sont présentés tableau III.

C'est donc la source d'aluminium qui règle les pressions partielles dans l'enceinte, indépendamment de la source de $\mathrm{SiO}$.

c) Enfin, on dégaze très rapidement $(10 \mathrm{mn})$ la source de fer et on commence l'évaporation simultanée Al-Fe-SiO. Durant cette évaporation, la composition des gaz résiduels varie entre celles données par la $3^{\mathrm{e}}$ et la $4^{\mathrm{e}}$ colonne du tableau III. La forte variation de la composition de l'atmosphère résiduelle vient essentiellement de l'oxyde de carbone ; le diagramme IV permet en effet de constater la relative stabilité des différents courants d'ions, excepté ceux des masses 28 et 12 .

IV. Conclusion. - Le dispositif d'évaporation mis au point permet la réalisation de couches multiples
Al-Fe-SiO. Le nombre maximal de couches peut atteindre 2000 pour un temps de manipulation de l'ordre de $4 \mathrm{~h}$ grâce à l'utilisation d'une source d'évaporation d'aluminium alimentée en continu dont la vitesse d'évaporation peut atteindre $500 \AA / \mathrm{s}$ à une distance de $10 \mathrm{~cm}$. Les échantillons obtenus présentent une section efficace d'absorption convenable en spectrométrie Mössbauer et les spectres obtenus [14], de très bonne qualité, sont parfaitement exploitables, même si on n'utilise pas du fer enrichi en isotope ${ }^{57} \mathrm{Fe}$.

Durant l'évaporation simultanée Al-Fe-SiO, l'atmosphère résiduelle de l'enceinte est constituée de $31 \%$ de vapeur d'eau, 31,5\% d'hydrogène et $13 \%$ d'oxyde de carbone, les autres gaz intervenant dans des proportions beaucoup plus faibles. Cette composition est déterminée par les gaz présents dans l'enceinte avant l'évaporation et ceux libérés par les sources d'évaporation d'une part, par l'action sur ces gaz des matériaux évaporés d'autre part.

C'est ainsi que la pression partielle de vapeur d'eau est initialement déterminée par l'atmosphère du Laboratoire et une fuite, mais les variations dues au dégazage des parois de l'enceinte se trouvent très fortement atténuées par l'effet getter de $\mathrm{SiO}$ et de $\mathrm{Al}$.

L'hydrogène provient essentiellement du creuset en tantale contenant $\mathrm{SiO}$; une certaine fraction vient de la réaction de $\mathrm{SiO}$ sur $\mathrm{H}_{2} \mathrm{O}$; enfin une dernière partie est libérée par la source d'aluminium. Ce gaz constitue toujours une fraction très importante de l'atmosphère résiduelle dès qu'une source d'évăporation ou un filament est chauffé sous vide.

Enfin, l'oxyde de carbone provient presque exclusivement de la source d'aluminium. Il constitue $50 \%$ de l'atmosphère résiduelle durant l'évaporation simultanée $\mathrm{Al}-\mathrm{SiO}$, mais sa pression partielle diminue fortement dès que l'évaporation simultanée $\mathrm{Al}-\mathrm{Fe}-\mathrm{SiO}$ a lieu. On peut donc logiquement se demander si le fer ne piège pas l'oxyde de carbone par effet getter.

Cette analyse des gaz résiduels durant les évaporations indique clairement qu'il y a une pollution des matériaux durant l'élaboration des échantillons : pol- 
lution certaine par la vapeur d'eau qui est fixée sur l'aluminium et la silice amorphe, pollution probable du fer par l'oxyde de carbone. Le risque d'avoir des composés de nature plus ou moins ionique (oxydes, hydroxydes,...) n'est donc pas à négliger. L'espoir de mesurer par effet Mössbauer les caractéristiques locales d'atomes adsorbés, vraiment métalliques, passe donc nécessairement par une amélioration des conditions expérimentales : augmentation du vide limite et de la vitesse de pompage dans l'enceinte d'évaporation.

Remerciements. - Ce travail a été réalisé dans le cadre du Laboratoire associé $n^{\circ} 155$ au Centre National de la Recherche Scientifique. Nous remercions la société Veeco qui nous a largement fait profiter de son assistance technique.

\section{Bibliographie}

[1] JANOT (C.), L'effet Mössbauer et ses applications. Masson éd., Paris, 1972.

[2] Honig (R. E.) et Kramer (D. A.), R. C. A. Review, 1969, 30, 285

[3] Klopfer (A.) et Schmidt (W.), Vacuum, 1960, 10, 363.

[4] American Petroleum Institute, Mass spectral Data (Carnegie Institute, Pittsburgh).

[5] Cleaver (J. S.) et Fiveash (P. N.), Vacuum, 1970, 20, 49.

[6] Wood Jr (G. M.) et Roenigk Jr (R. J.), J. Vac. Sci. and Techn., 1969, 6, 871.

[7] Wagener (J. W.) et Mark (P. T.), J. Appl. Phys., 1957, 28, 1029.
[8] Pitt (K. E. G.) et Howard (A. J.), Vacuum, 1968, 18, 517.

[9] Allam (D. S.) et Pitt (K. E. G.), Thin Solid Films, 1967, 1, 245.

[10] Hill (A. E.) et Hoffman (G. R.), Br. J. Appl. Phys., 1967, 18, 13.

[11] Priest (J.), Caswell (H. L.) et Budo (Y.), Vacuum, 1962, 12, 301.

[12] Beitel (G. A.), Techn. Rep. NASA CR-111829, 1971.

[13] Norton (F. J.) et Marshall (A. L.), Trans. AIME Inst. Metals Div., 1944, 156, 351.

[14] Janot (C.), Marchal (G.), Piecuch (M.) et ScherRer (H.), C. R. Acad. Sci. Paris, 1971, 273, 399. 